Pacific

Journal of

Mathematics

SPACES OF INTEGRABLE FUNCTIONS WITH RESPECT TO VECTOR MEASURES OF CONVEX RANGE AND FACTORIZATION OF OPERATORS FROM $L_{p}$-SPACES E.A. SÁNCHEZ PÉREZ 


\title{
SPACES OF INTEGRABLE FUNCTIONS WITH RESPECT TO VECTOR MEASURES OF CONVEX RANGE AND FACTORIZATION OF OPERATORS FROM $L_{p}$-SPACES
}

\author{
E.A. SÁnChez PÉRez
}

If $G$ is a Banach space valued measure whose range range is convex, we consider the space of $G$-integrable functions. We also establish a factorization result for operators from $L_{p}(\mu)$ through $L_{1}(G)$. We apply these results in order to obtain a description of the range of $p^{\prime}$-summing operators from $L_{p^{-}}$ spaces.

\section{Introduction and notation.}

Let $(\Omega, \Sigma)$ be a measurable space and let $X$ be a Banach space. Throughout this paper $G$ will be a countably additive vector measure $G: \Sigma \rightarrow X$. Consider the space $L_{1}(G)$ of (classes of real) $G$-integrable functions, following the definition of Bartle, Dunford and Schwartz [1] and Lewis [9]. The properties of this space have been studied by Kluvánek and Knowles [7], Okada [10] and Curbera [2]. In the first part of this paper (Section 1) we investigate the relation between the convexity of the range of $G$ and the structure of the space $L_{1}(G)$. We use an alternate definition of the norm of $L_{1}(G)$ that is obtained by identifying each function $f \in L_{1}(G)$ with an operator from an $L_{\infty}$ space. In the second part (Section 2), we apply these ideas to obtain several properties of operators from an $L_{p}$ space to a Banach space. In particular, we use the results of Dinculeanu that relates vector measures and operators from $L_{p}$ spaces (see [6]) to obtain a factorization theorem. As an application, we also show a description of the range of $p^{\prime}$-summing operators from $L_{p}$ spaces.

We use well-known results about general Vector Measure Theory (see [5]). The notation is standard. If $x^{\prime} \in X^{\prime},\left|x^{\prime} G\right|$ is the variation of the scalar measure $x^{\prime} G$ defined by $x^{\prime} G(A):=\left\langle G(A), x^{\prime}\right\rangle$. The semivariation of $G$ in a set $A \in \Sigma$ is given by $\|G\|(A)=\sup \left\{\left|x^{\prime} G\right|(A): x^{\prime} \in B_{X^{\prime}}\right\}$. We write $\chi_{A}$ for the characteristic function of $A \in \Sigma$ and $A^{c}$ for $\Omega \backslash A$. If $1 \leq p<\infty, p^{\prime}$ is the (extended) real number that satisfies $1 / p+1 / p^{\prime}=1$. The space of linear and continuous operators between the Banach spaces $Y$ and $X$ is denoted by $L(Y, X)$.

A measurable real function defined on $\Omega$ is $G$-integrable if it is $x^{\prime} G$ integrable for each $x^{\prime} \in X^{\prime}$, and for every $A \in \Sigma$ there is an element $\int_{A} f d G$ 
of $X$ such that $\left\langle\int_{A} f d G, x^{\prime}\right\rangle=\int_{A} f d x^{\prime} G$ for every $x^{\prime} \in X^{\prime}([\mathbf{9}])$. The Banach lattice $L_{1}(G)$ is the space of all the (classes of) $G$-integrable functions with the $\|G\|$-almost everywhere order, endowed with the norm

$$
\|f\|_{G}:=\sup \left\{\int_{\Omega}|f| d\left|x^{\prime} G\right|: x^{\prime} \in B_{X^{\prime}}\right\}, \quad f \in L_{1}(G) .
$$

The expression $|\|f\||_{G}:=\sup _{A \in \Sigma}\left\|\int_{A} f d G\right\|, f \in L_{1}(G)$, provides an equivalent norm that satisfies the inequalities $\left|\|f\|\left\|_{G} \leq\right\| f\left\|_{G} \leq 2 \mid\right\| f \|_{G}\right.$. However, although the norm $\mid\|\cdot\| \|_{G}$ appears in the literature, it does not seem to be a natural one. The integration operator $I_{G}: L_{1}(G) \rightarrow X$ is defined by $I_{G}(f):=\int_{\Omega} f d G, f \in L_{1}(G)$ (see $[\mathbf{1 1}]$ ). Let $\mu$ be a finite measure that controls $G$ (see [5] Ch. IX). Integration with respect to $G$ also gives a continuous linear map $T_{G} \in L\left(L_{\infty}(\mu), X\right)$. Let $S(\mu)$ be the set of (classes of) simple functions (that are equal $\mu$-a.e.).

Lemma 1. The following formula gives the $L_{\infty}$-norm on $S(\mu)$;

$$
\|f\|_{\infty}=\inf \left\{\sum_{i=1}^{n}\left|\lambda_{i}\right|: f=\sum_{i=1}^{n} \lambda_{i}\left(\chi_{A_{i}}-\chi_{A_{i}^{c}}\right), \quad A_{i} \in \Sigma\right\}, \quad f \in S(\mu) .
$$

Proof. Let $f \in S(\mu)$. Then we can find a finite family of disjoint measurable subsets $\left(A_{i}\right)_{i=1}^{n}$ and real numbers $(\lambda)_{i=1}^{n}$ such that $f=\sum_{i=1}^{n} \lambda_{i} \chi_{A_{i}}$. Thus, the function $f$ belongs to the subspace of $L_{\infty}(\mu)$ that is obtained by the restriction of this space to the finite subalgebra generated by $\left\{A_{i}: i=\right.$ $1, \ldots, n\}$. This is a finite dimensional $L_{\infty}$ space, and then is isometric to $l_{\infty}^{n}$. Since the extreme points of the unit ball on $l_{\infty}^{n}$ are the elements of $\{-1,1\}^{n}$, we obtain the result as a direct consequence of the Krein-Milman Theorem.

Definition 2. Let $\mu$ be a finite control measure for $G$. For every $f \in L_{1}(G)$, we define $\|f\|_{G \infty}:=\sup _{g \in B_{L_{\infty}(\mu)}}\left\|\int_{\Omega} f g d G\right\|$.

Note that the definition of $\|\cdot\|_{G \infty}$ does not depend on the particular control measure $\mu$. This is a consequence of the following simple argument. Each function $f \in L_{1}(G)$ defines the countably additive vector measure $G_{f}$ and the semivariation of this measure gives an equivalent norm for the space $L_{1}(\lambda)$ (see [9] or [2]). Theorem 13 of [5, Ch. I] for the case of a finite measure $\mu$ defined over the $\sigma$-algebra $\Sigma$ establishes the equality between the norm of the operator $T_{G_{f}}$ and the semivariation of $G_{f}$. A direct application of the particular representation of the norm of the functions of $L_{\infty}(\mu)$ given in Lemma 1 leads to the following result, that simplifies the arguments related to the convexity of $G$.

Proposition 3. For every $f \in L_{1}(G)$,

$$
\|f\|_{G}=\|f\|_{G \infty}=\sup _{A \in \Sigma}\left\|\int_{\Omega} f\left(\chi_{A}-\chi_{A^{c}}\right) d G\right\| .
$$


Proof. First note that if $x^{\prime} \in X^{\prime}$ and $A \in \Sigma$ the variation of the scalar measure $x^{\prime} G$ on $A$ is given by

$$
\left|x^{\prime} G\right|(A)=\sup \left\{\left\langle G(A \cap B), x^{\prime}\right\rangle-\left\langle G\left(A \cap B^{c}\right), x^{\prime}\right\rangle: B \in \Sigma\right\} .
$$

Let us denote $\sup _{A \in \Sigma}\left\|\int_{\Omega} f\left(\chi_{A}-\chi_{A^{c}}\right) d G\right\|$ by $\|f\|_{s}$ for every $f \in L_{1}(G)$. Let $f$ be a simple function and let $\epsilon>0$. Then a direct calculation using the above representation of $\left|x^{\prime} G\right|$ shows that there is a set $B \in \Sigma$ such that the function $h:=\chi_{B}-\chi_{B^{c}}$ satisfies $\|f\|_{G} \leq\left\langle\int_{\Omega} f h d G, x^{\prime}\right\rangle+\epsilon$. This and the density of the simple functions in $L_{1}(G)$ imply the inequalities $\|f\|_{G} \leq$ $\|f\|_{s} \leq\|f\|_{G \infty}$ for every $f \in L_{1}(G)$. Since the inequality $\|f\|_{s} \leq\|f\|_{G}$ is obvious and Lemma 1 implies $\|f\|_{G \infty} \leq\|f\|_{s}$ for every $f \in L_{1}(G)$, we obtain the result.

Let $\mu$ be a Rybakov control measure for $G$ (see [5, Ch. IX.2]). The main known result that relates the structure of the space $L_{\infty}(\mu)$ with the convexity and weak compactness of the class of sets $\{G(A \cap B): B \in \Sigma\}, A \in \Sigma$, is the following theorem due to Knowles (see Theorem 4 and Corollary 7 of $[\boldsymbol{5}$, Ch. IX.1]).

Theorem 4. The following are equivalent:

1) If $0 \neq f \in L_{\infty}(\mu)$, there is a function $g \in L_{\infty}(\mu)$ such that $\|f g\|_{\infty}>0$ but $\int_{\Omega} f g d G=0$.

2) For each $A \in \Sigma,\{G(A \cap B): B \in \Sigma\}$ is a weakly compact convex set in $X$.

3) For each $A \in \Sigma$ there is a set $B \in \Sigma$ such that $G(A \cap B)=\frac{G(A)}{2}$.

We will say that a vector measure $G$ that satisfies any one of the statements of Theorem 4 is a $\Sigma$-weakly compact convex vector measure. If $G$ is such a measure, $A \in \Sigma$ and $G(A) \neq 0$, we can define the following tree of subsets. An application of Statement 3) of Theorem 4 gives a set $A_{1}^{1} \in \Sigma$ such that $A_{1}^{1} \subset A$ and $G\left(A_{1}^{1}\right)=\frac{G(A)}{2}$. Then, if we define the set $A_{1}^{2} \in \Sigma$ as $A_{1}^{2}=A-A_{1}^{1}$, we obtain $G\left(A_{1}^{1}\right)=\frac{G(A)}{2}=G\left(A_{1}^{2}\right)$. Following the same procedure for $G\left(A_{1}^{1}\right)$ and $G\left(A_{1}^{2}\right)$, we obtain four subsets $G\left(A_{2}^{i}\right), i=1, \ldots, 4$, such that $A_{2}^{1} \cup A_{2}^{2}=A_{1}^{1}, A_{2}^{3} \cup A_{2}^{4}=A_{1}^{2}$ and $G\left(A_{2}^{i}\right)=\frac{G(A)}{4}$ for each $i$. We may continue in this way in order to obtain a tree of sets $\left\{A_{n}^{i}\right\}$ such that:

1) For each natural number $n,\left\{A_{n}^{i}\right\}$ is a partition of $A$ and for every $k=1, \ldots, 2^{n-1}, A_{n}^{2 k-1} \cup A_{n}^{2 k}=A_{n-1}^{k}$.

2) For each natural number $n$ and every $i=1, \ldots, 2^{n}, G\left(A_{n}^{i}\right)=\frac{G(A)}{2^{n}}$.

This sequence of subsets leads to a $\sigma$-subalgebra $\Sigma_{A}$ of $\Sigma$, the restriction of $G$ to which is one dimensional and equivalent to the Lebesgue measure on $[0,1]$. We thus have: 
Corollary 5. In the notation above, denote by $G_{A}$ the restriction of $G$ to $\Sigma_{A}$. Then $\left(L_{1}\left(G_{A}\right),\|\cdot\|_{G_{A}}\right)$ is isometric to $L_{1}(0,1)$.

Remark 6. It is clear that $\|f\|_{G_{A}} \leq\|f\|_{G}$ for every function $f \in L_{1}\left(G_{A}\right)$. If $A \in \Sigma$ and there is a constant $K_{A}$ such that $\|f\|_{G} \leq K_{A}\|f\|_{G_{A}}$ for every function $f \in L_{1}\left(G_{A}\right)$ we can identify the space $L_{1}\left(G_{A}\right)$ with a subspace of $L_{1}(G)$. For instance, this is the case when $G$ is defined on a Banach lattice and integration with respect to $G$ gives a positive operator. The above construction yields a lot of information about the structure of the function spaces $L_{1}\left(G_{A}\right)$, since the structure of $L_{1}(0,1)$ is very well-known (see Th. 7 of $[8$, Ch. 6.17]).

\section{A factorization theorem.}

Let $\mu$ be a (countably additive purely nonatomic) finite measure. In this section we obtain several properties of the range of the operators of $L\left(L_{p}(\mu), X\right)$ related to the Uhl Theorem about the relative compactness of the range of a vector measure $G$. Through this section we consider the norm \|\|$_{G \infty}$ for $L_{1}(G)$. The results of Dinculeanu about vector measure integration (see [6]) allow to a factorization theorem for these operators. Let $P(\Sigma)$ be the class of all the finite partitions of $\Omega$ in $\Sigma$. The following definition can be found in $[6$, Ch. II.13].

Definition 7. If $\mu$ be a finite control measure for $G$ and $1 \leq p<\infty$, the $p$-semi-variation of $G$ in $A \in \Sigma$ is defined by

$$
\|G\|_{p}^{s}(A):=\sup _{\left\|x^{\prime}\right\| \leq 1,\left\{A_{i}\right\}_{i=1}^{n} \in P(\Sigma)}\left(\sum_{i=1}^{n} \frac{\left|\left\langle G\left(A_{i} \cap A\right), x^{\prime}\right\rangle\right|^{p}}{\mu\left(A_{i} \cap A\right)^{\frac{p}{p^{\prime}}}}\right)^{\frac{1}{p}} .
$$

We denote by $M_{p}^{s}(\mu, X)$ the space of all the $X$-valued vector measures of finite $p$-semi-variation $\|G\|_{p}^{s}:=\|G\|_{p}^{s}(\Omega)$. If $T \in L\left(L_{p}(\mu), X\right)$, we write $G_{T}$ for the vector measure given by $G_{T}(A):=T\left(\chi_{A}\right), A \in \Sigma$. Conversely, if $G: \Sigma \rightarrow X$ is a vector measure and $\mu$ controls $G$, we denote by $T_{G}$ the linear map defined on $S(\mu)$ by $T_{G}\left(\chi_{A}\right):=G(A)$. We will use the same notation for this map if it is possible to extend it to the space $L_{p}(\mu)$. In our context the general result given in [6, Ch. II.13, Th. 1] can be written as follows. We obtain Theorem 11 as a consequence.

Theorem 8. $M_{p^{\prime}}^{s}(\mu, X)$ is isomorphic to the space $L\left(L_{p}(\mu), X\right)$.

Theorem 9. Let $1 \leq p<\infty$ and $T \in L\left(L_{p}(\mu), X\right)$. Then it can be factored through $L_{1}\left(G_{T}\right)$ as

$$
L_{p}(\mu) \stackrel{I}{\rightarrow} L_{1}\left(G_{T}\right) \stackrel{I_{G_{T}}}{\rightarrow} X
$$

where $I(f):=\bar{f}$, if $\bar{f}$ is the class of $f$ in $L_{1}\left(G_{T}\right)$, and $I_{G_{T}}(\bar{f})=\int_{\Omega} \bar{f} d G_{T}$. Moreover, $\left\|G_{T}\right\|_{p^{\prime}}^{s} \leq\|T\| \leq\|I\| \leq 2\left\|G_{T}\right\|_{p^{\prime}}^{s}$. 
Proof. We need to show that $\|f\|_{G \infty} \leq\|T\|$ if $\|f\|_{L_{p}} \leq 1$. By Proposition 3, it is enough to estimate $\left\|\int_{\Omega} f g d G_{T}\right\|$, where $\|g\|_{L_{\infty}} \leq 1$. But this integral is just $T(f g)$. Since $\|f g\|_{L_{p}} \leq 1$, we obtain $\|T(f g)\| \leq\|T\|$, as required. The inequalities between the norms of the operators and the $p^{\prime}$-semi-variation can be obtained by mean of a direct calculation following the arguments given above and the ones that can be found in [6].

The properties of the operator $T: L_{p}(\mu) \rightarrow X$ and the space $X$ determine the structure of the factorization space $L_{1}\left(T_{G}\right)$. For example, if the measure $G_{T}$ is $\Sigma$-weakly compact convex and $A \in \Sigma$, the restriction of the operator to the subspace of $L_{p}(\mu)$ generated by $\Sigma_{A}$ factorizes through $L_{1}(0,1)$, as a consequence of the arguments given in Section 1. Nowadays, a lot of properties of the space $L_{1}(G)$ that may be applied in our context are known (see $[\mathbf{1 0}],[\mathbf{2}]$ and $[\mathbf{3}]$ ). We present an example that is a consequence of $[\mathbf{2}$, Th. 3].

Corollary 10. Let $1 \leq p<\infty$ and let $X$ be a cotype 2 Banach space. Consider an operator $T: L_{p}(\mu) \rightarrow X$. If for every partition $\left(A_{n}\right)_{1}^{\infty}$ the sequence $\left(\frac{T\left(\chi_{A_{n}}\right)}{\left\|G_{T}\right\|\left(A_{n}\right)}\right)_{1}^{\infty}$ is 2-lacunary in $X$, then $T$ factors through a space that is order isomorphic to a Hilbert space.

To finish this paper, we apply the above results to obtain a description of the range of the $p^{\prime}$-summing operators from $L_{p}(\mu)$ to $X$. The reader can find information about the operator ideals of p-summing operators $\left(P_{p}, \Pi_{p}\right)$ in [4] and [12]. Let $C$ be a subset of a Banach space $Y$ and let $T \in L(Y, X)$. We say that the range of $T$ is approximable by $C$ if the set $\{\lambda T(y): \lambda \in$ $R, y \in C\}$ is dense in the range of $T$. Let $B_{0}:=\left\{\chi_{A}-\chi_{A^{c}}: A \in \Sigma\right\}$.

Theorem 11. Let $1<p<\infty$ and $\mu$ be a nonatomic probability measure. Let $X$ be a Banach space with the Radon-Nikodym property and let $R \in$ $L\left(L_{p}(\mu), X\right)$. If $R$ is $p^{\prime}$-summing, then the range of $R$ is approximable by $B_{0}$.

Proof. First we claim that the composition of the operators $T: L_{p}(\mu) \rightarrow Y$ and $S: Y \rightarrow X$, if $T$ is continuous and $S$ is $p^{\prime}$-summing, defines a countably additive vector measure $G_{S T}$ of bounded variation. Since $G_{S T}(A)=$ $S T\left(\chi_{A}\right)$, we get $\left\|G_{S T}(A)\right\| \leq\|S T\| \mu(A)^{\frac{1}{p}}$ and then $G_{S T}$ is countably additive. Let $\left\{A_{i}\right\}_{i=1}^{n} \in P(\Sigma)$. Then

$$
\begin{aligned}
\sum_{i=1}^{n}\left\|S T\left(\chi_{A_{i}}\right)\right\| & =\sum_{i=1}^{n} \mu\left(A_{i}\right)^{\frac{1}{p}} \frac{\left\|S T\left(\chi_{A_{i}}\right)\right\|}{\mu\left(A_{i}\right)^{\frac{1}{p}}} \\
& \leq\left(\sum_{i=1}^{n} \frac{\left\|S T\left(\chi_{A_{i}}\right)\right\|^{p^{\prime}}}{\mu\left(A_{i}\right)^{\frac{p^{\prime}}{p}}}\right)^{\frac{1}{p^{\prime}}}
\end{aligned}
$$




$$
\begin{aligned}
& \leq \Pi_{p^{\prime}}(S) \sup _{\left\|x^{\prime}\right\|_{Y} \leq 1}\left(\sum_{i=1}^{n} \frac{\left|\left\langle T\left(\chi_{A_{i}}\right), x^{\prime}\right\rangle\right|^{p^{\prime}}}{\mu\left(A_{i}\right)^{\frac{p^{\prime}}{p}}}\right)^{\frac{1}{p^{\prime}}} \\
& \leq \Pi_{p^{\prime}}(S)\left\|G_{T}\right\|_{p^{\prime}}^{s} .
\end{aligned}
$$

Since $T$ is a continuous map, Theorem 9 gives that $\left\|G_{T}\right\|_{p^{\prime}}^{s}<\infty$. Now, suppose that $X$ has the Radon-Nikodym property. Since $\mu$ is nonatomic, so is $G_{S T}$. An application of Uhl Theorem (see [5] Ch. IX.1) gives the convexity of the closure of the range of $G_{S T}$. Since

$$
\overline{\left\{G_{S T}(A): A \in \Sigma\right\}}=\frac{1}{2} G_{S T}(\Omega)+\frac{1}{2} \overline{S T\left(B_{0}\right)},
$$

and $B_{L_{\infty}(\mu)}=\overline{c o\left(B_{0}\right)}$ as a consequence of Lemma 1, we obtain $\overline{S T\left(B_{0}\right)}=$ $\overline{S T\left(B_{L_{\infty}(\mu)}\right)}$. This obviously proves that the range of $S T$ is approximable by $B_{0}$. To finish the proof it is enough to consider the factorization of $R$ as $R=R I$, where $I$ is the identity map in $L_{p}(\mu)$.

It is possible to find several applications of this theorem in the context of the Operator Ideals Theory. Obviously, it also holds for each $p^{\prime}$-integral operator $T \in L\left(L_{p}(\mu), X\right)$. The same argument can be applied for $(p, q)$ summing operators. In the context of the Hilbert spaces, we may obtain that each Hilbert-Schmidt operator satisfies that its range is approximable by $B_{0}$.

Acknowledgements. The author thanks the referee for his thorough and careful reading of the manuscript and gratefully acknowledges his many helpful suggestions.

\section{References}

[1] R.G. Bartle, N. Dunford and J. Schwartz, Weak compactness and vector measures, Canad. J. Math., 7 (1955) 289-305, MR 16,1123c, Zbl 0068.09301.

[2] G.P. Curbera, Banach space properties of $L^{1}$ of a vector measure, Proc. Am. Math. Soc., 123 (1995), 3797-3806, MR 96b:46060, Zbl 0848.46015.

[3] W When $L^{1}$ of a vector measure is an AL-space, Pacific J. Math., 162(2) (1994), 287-303, MR 94k:46070, Zbl 0791.46021.

[4] J. Diestel, H. Jarchow and A. Tonge, Absolutely Summing Operators, Cam. St. Ad. Math., 43, Cambridge University Press, Cambridge, 1995, MR 96i:46001, Zbl 0855.47016 .

[5] J. Diestel and J.J. Uhl, Vector Measures, Math. Surveys, 15, Amer. Math. Soc. Providence, 1977, MR 56 \#12216, Zbl 0369.46039.

[6] N. Dinculeanu, Vector Measures, Pergamon Press, Berlin, 1967, MR 34 \#6011b, Zbl 0142.10502.

[7] I. Kluvánek and G. Knowles, Vector Measures and Control Systems, North-Holland, Amsterdam, 1975, MR 58 \#17033, Zbl 0316.46043. 
[8] H.E. Lacey, The Isometric Theory of Classical Banach Spaces, Springer, Berlin, 1974, MR 58 \#12308, Zbl 0285.46024.

[9] D.R. Lewis, Integration with respect to vector measures, Pacific J. Math., 33 (1970), 157-165, MR 41 \#3706, Zbl 0195.14303.

[10] S. Okada, The dual space of $L^{1}(\mu)$ of a vector measure $\mu$, J. Math. Anal. Appl., 177 (1993), 583-599, MR 94m:46050, Zbl 0804.46049.

[11] S. Okada and W.J. Ricker, The range of the integration map of a vector measure, Arch. Math., 64 (1995), 512-522, MR 96e:46057, Zbl 0832.28014.

[12] A. Pietsch, Operator Ideals, North Holland Math. Library, North-Holland, Amsterdam, New York, 1980, MR 81j:47001, Zbl 0434.47030.

Received December 10, 1999 and revised April 18, 2002.

Departamento de Matemática Aplicada

Universidad Politécnica de VAlencia

46071 VALENCIA, SPAin

E-mail address: easancpe@mat.upv.es 